\title{
Complex dielectric modulus and relaxation response at low microwave frequency region of dielectric ceramic $\mathrm{Ba}_{6-3 x} \mathrm{Nd}_{8+2 x} \mathrm{Ti}_{18} \mathrm{O}_{54}$
}

\author{
Chian Heng Lee*,*, Jumiah Hassan ${ }^{* \dagger}$, Mansor Hashim ${ }^{\dagger}$, \\ Raba'ah Syahidah Aziz ${ }^{*} \dagger$ and Norlaily Mohd Saiden* \\ *Department of Physics, Universiti Putra Malaysia 43400 Serdang \\ Selangor, Malaysia \\ $\dagger^{\dagger}$ Institute of Advanced Technology \\ Universiti Putra Malaysia 43400 Serdang \\ Selangor, Malaysia \\ tchianheng16@gmail.com
}

Received 7 July 2014; Revised 28 October 2014; Accepted 17 November 2014; Published 2 December 2014

\begin{abstract}
The desirable characteristics of $\mathrm{Ba}_{6-3 x} \mathrm{Nd}_{8+2 x} \mathrm{Ti}_{18} \mathrm{O}_{54}$ include high dielectric constant, low loss tangent, and high quality factor developed a new field for electronic applications. The microwave dielectric properties of $\mathrm{Ba}_{6-3 x} \mathrm{Nd}_{8+2 x} \mathrm{Ti}_{18} \mathrm{O}_{54}$, with $x=0.15$ ceramics at different sintering temperatures $\left(600-1300^{\circ} \mathrm{C}\right)$ were investigated. The phenomenon of polarization produced by the applied electric field was studied. The dielectric properties with respect to frequency from $1 \mathrm{MHz}$ to $1.5 \mathrm{GHz}$ were measured using Impedance Analyzer, and the results were compared and analyzed. The highest dielectric permittivity and lowest loss factor were defined among the samples. The complex dielectric modulus was evaluated from the measured parameters of dielectric measurement in the same frequency range, and used to differentiate the contribution of grain and grain boundary.
\end{abstract}

Keywords: Ceramic; sintering; dielectric response; electrochemical impedance spectroscopy.

\section{Introduction}

Materials that have excellent microwave dielectric properties have many applications for use in mobile and satellite communication systems, antennas, oscillators, filters, wireless communications, and sensors. ${ }^{1-3}$ For microwave electronic devices, the main characteristics of the material include high dielectric permittivity and high quality factor. ${ }^{4} \mathrm{Li}$ et $a l .{ }^{5}$ reported that $\left(\mathrm{Mg}_{0.95} \mathrm{Zn}_{0.05}\right)_{1.8} \mathrm{Ti}_{1.1} \mathrm{O}_{4}$ doped with $\mathrm{CaTiO}_{3}$ ceramic improved the microwave dielectric properties. Thomas et al. ${ }^{6}$ found the dielectric constant at $1 \mathrm{MHz}$ for $\mathrm{Ca}_{4} \mathrm{La}_{6}\left(\mathrm{SiO}_{4}\right)_{4}$ $\left(\mathrm{PO}_{4}\right)_{2} \mathrm{O}_{2}$ with different isovalent substitutions of $\mathrm{Sr}^{2+}, \mathrm{Pr}^{3+}$, $\left(\mathrm{GeO}_{4}\right)^{4-}$, and $\left(\mathrm{VO}_{4}\right)^{3-}$ to be around 14. Huang et $\mathrm{al}^{7}{ }^{7}$ promoted a high $Q$ dielectric ceramic $\left(\mathrm{Mg}_{0.95} \mathrm{Zn}_{0.05}\right)_{4} \mathrm{Ta}_{2} \mathrm{O}_{9}$ with dielectric constant of 12.67. In the previous paper, ${ }^{8}$ evolution of microstructure of $\mathrm{Ba}_{6-3 x} \mathrm{Nd}_{8+2 x} \mathrm{Ti}_{18} \mathrm{O}_{54}$ with $x=0.15$ was studied. These types of materials are currently widely used in the development of microwave telecommunication technology due to their high dielectric constant. ${ }^{9-12}$ The cations in the solid solutions are important to the dielectric properties of the compounds. The chemical formula $\mathrm{Ba}_{6-3 x} \mathrm{R}_{8+2 x} \mathrm{Ti}_{18} \mathrm{O}_{54}$ where $R$ is rare earth element shows the effect on the replacement of Ba with $\mathrm{Nd}$ elements. In this case, the atomic ratio of titanium to oxygen is $1: 3$ is similar to perovskite structure $\mathrm{ABX}_{3} \cdot{ }^{13,14}$ Therefore, it also exhibits piezoelectric behavior that can be used as transducer.

\section{Experimental Methods}

The starting materials used in this research were high purity and nano size $\mathrm{BaCO}_{3}(99.9 \%$, Nanoshel below $80 \mathrm{~nm})$, $\mathrm{Nd}_{2} \mathrm{O}_{3}$ (99.9\%, US Research Nanomaterials $30-45 \mathrm{~nm}$ ), and $\mathrm{TiO}_{2}(100 \%$, US Research Nanomaterials $50 \mathrm{~nm}$,) powders. The samples were prepared by magnetic stirring method. The raw materials were weighed by Shimadzu Analytical Balance AY220 according to compositional formula $\mathrm{Ba}_{6-3 x}$ $\mathrm{Nd}_{8+2 x} \mathrm{Ti}_{18} \mathrm{O}_{54}$ with $x=0.15$, and mixed with ethanol using WiseStir MSH-20D Hotplate Magnetic Stirrer. The liquid mixtures were milled for $24 \mathrm{~h}$ at $30^{\circ} \mathrm{C}$ with milling speed of $900 \mathrm{rpm}$ to get homogenous solutions. The liquid mixtures were dried for $24 \mathrm{~h}$. The resulting powders were then compacted into pellets using Carver manual pellet press with pressure of $216 \mathrm{MPa}$. Each pellet has diameter of $17 \mathrm{~mm}$ and thickness of $2.8 \mathrm{~mm}$. The final pellets were sintered for $3 \mathrm{~h}$ in air in a programmable furnace at different sintering temperatures in the range from $600^{\circ} \mathrm{C}$ to $1300^{\circ} \mathrm{C}$ at $100^{\circ} \mathrm{C}$ intervals. The dielectric properties of the samples were measured by Impedance Analyzer (HP Model 4291B) from $1 \mathrm{MHz}$ to $1.5 \mathrm{GHz}$ at room temperature. The sample was measured by placing it between two conducting plates with applied electric field. The measured dielectric properties of the materials can be represented in terms of complex dielectric permittivity $\varepsilon^{*}=\varepsilon^{\prime}-j \varepsilon^{\prime \prime}$, where $\varepsilon^{\prime}$ is the real part of

This is an Open Access article published by World Scientific Publishing Company. It is distributed under the terms of the Creative Commons Attribution 3.0 (CC-BY) License. Further distribution of this work is permitted, provided the original work is properly cited. 
dielectric permittivity, and $\varepsilon^{\prime \prime}$ is imaginary part of dielectric permittivity or dielectric loss factor. The complex modulus analysis was used to investigate the contribution of grains and grain boundary of the sample in the electrical response.

\section{Results and Discussion}

\subsection{Dielectric permittivity of BNT ceramics}

The dielectric permittivity of barium neodymium titanate ceramic with compositional formula $\mathrm{Ba}_{6-3 x} \mathrm{Nd}_{8+2 x} \mathrm{Ti}_{18} \mathrm{O}_{54}$ at $x=0.15$ as a function of frequency for all sintering temperatures was shown in Fig. 1. The results show that dielectric permittivity of $600^{\circ} \mathrm{C}, 700^{\circ} \mathrm{C}$, and $800^{\circ} \mathrm{C}$ sintered ceramics exhibited strongly dispersive behavior at low frequency region. Initially, the dielectric permittivity decreases with increasing of sintering temperatures. The high dielectric permittivity for these sintering temperatures was also confirmed by X-ray diffraction (XRD) results due to the presence of $\mathrm{BaTiO}_{3}$ phase which has high dielectric permittivity at low frequency region. The XRD result for all sintering temperatures was revealed in Fig. 2 that have been published in previous work. ${ }^{8}$ Another reason that made the dielectric permittivity became higher at low frequency might be the occurrence of electrode polarization between the surface of the sample and the electrode itself or Maxwell-Wagner polarization. ${ }^{15-20}$ As electric field is applied to the sample, the charges were randomly accumulated on the sample, and created the depletion area that could increase the dielectric permittivity. However, the dielectric permittivity decreases as frequency increased due to the dipoles not being able to follow the alternating electric field. Dielectric properties of barium neodymium titanate (BNT) ceramic at $900^{\circ} \mathrm{C}$ sintering temperature shows a slight decrease from low frequency to high frequency region. The slight difference in the dielectric range can be considered as the formation of $\mathrm{Nd}_{2} \mathrm{Ti}_{2} \mathrm{O}_{7}$ phase and decomposed of $\mathrm{BaCO}_{3}$ phase in the ceramic. The dielectric permittivity shows more stability and independent

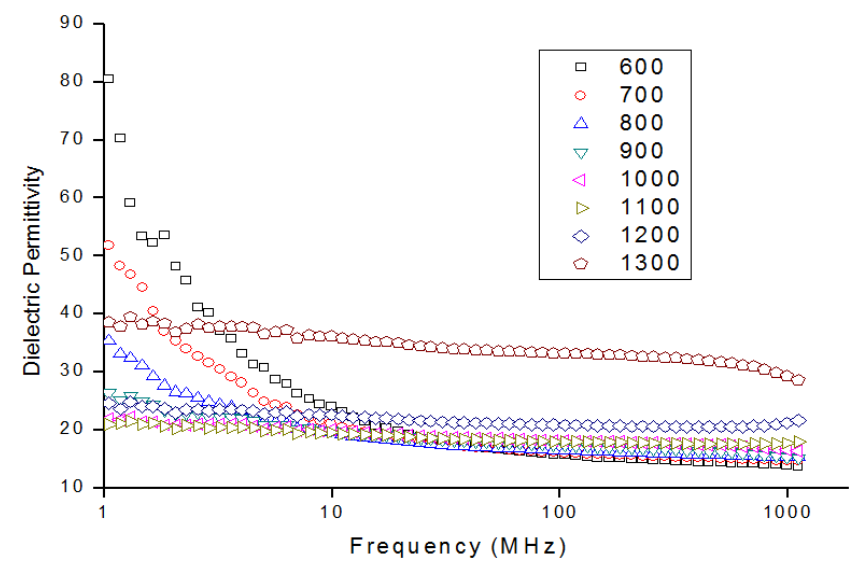

Fig. 1. Dielectric permittivity as a function of frequency for all sintering temperatures.

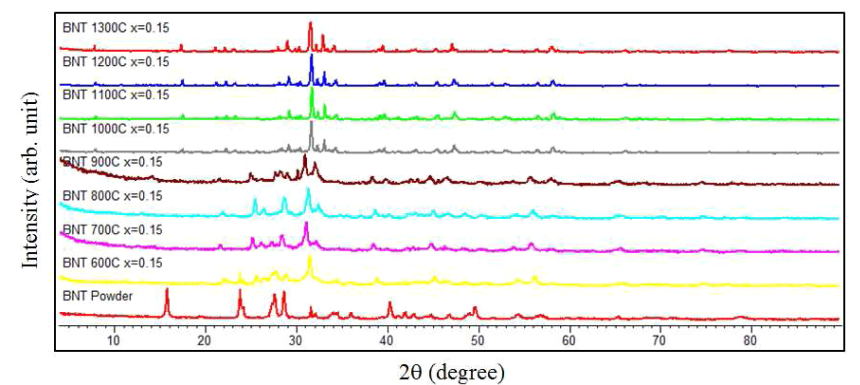

Fig. 2. XRD Pattern of BNT ceramic with $x=0.15$ for all sintering temperatures. $^{8}$

of frequency from $1000^{\circ} \mathrm{C}$ to $1300^{\circ} \mathrm{C}$ due to the formation of $\mathrm{BaNd}_{2} \mathrm{Ti}_{5} \mathrm{O}_{14}$ phase. The dielectric permittivity of BNT ceramic decreases from $1000^{\circ} \mathrm{C}$ to $1100^{\circ} \mathrm{C}$ due to the $\mathrm{XRD}$ pattern shifting to larger $2 \theta$ positions. ${ }^{8}$ BNT ceramic at $1100^{\circ} \mathrm{C}$ has the lowest dielectric permittivity among the samples. However, the dielectric permittivity shows another trend that increases again as sintering temperature increased from $1100^{\circ} \mathrm{C}$. The increment of dielectric permittivity at higher sintering temperature can be considered as the peak shifting to lower value of $2 \theta$ positions. This result also matched with the density measurement indicating higher density of the BNT ceramic and larger dielectric permittivity. It can be concluded that dielectric permittivity of the formed BNT ceramic is directly proportional to the density and sintering temperature. The dielectric relaxation strength is more significant for low sintered ceramics, and decreases as sintering temperature increases because there is only $\varepsilon_{\infty}$ that can be observed for high sintered ceramics in this frequency region. Figure 3 shows the dielectric permittivity of BNT ceramic as a function of sintering temperature at selected frequency. The results show the dielectric permittivity at $1 \mathrm{MHz}$ decreases exponentially from low sintering temperature to $1000^{\circ} \mathrm{C}$ indicating the decrease in electrode polarization as sintering temperature increases. One of the interesting findings is the broader peak of low sintered BNT ceramics in the XRD

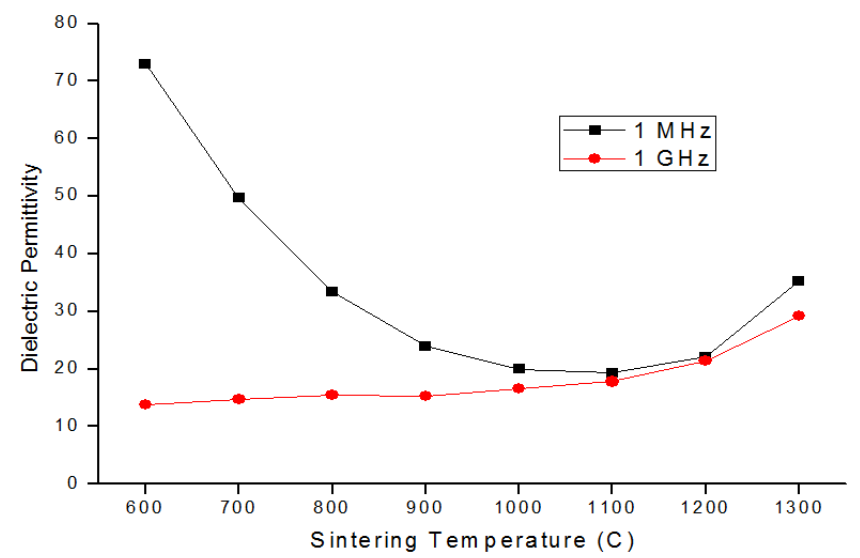

Fig. 3. Dielectric permittivity as a function of sintering temperatures at frequency of $1 \mathrm{MHz}$ and $1 \mathrm{GHz}$. 
pattern showing probably the occurrence of interfacial polarization at low microwave frequency region normally due to the existence of multiple phases in the ceramics. The main phase of the ceramics belongs to $\mathrm{BaTiO}_{3}$ phase which is the main reason for the polarization to occur. When sintered at high temperature, $\mathrm{Nd}^{3+}$ ions become more dominant and diffused into A1 site of the tungsten bronze structure. Replacement of $\mathrm{Nd}^{3+}$ ions successfully help to minimize the dielectric relaxation strength in the applied frequency region. On the other hand, the dielectric permittivity of ceramic at $1 \mathrm{GHz}$ basically is related to orientational polarization inside the material. It increases linearly with sintering temperature indicating higher sintering temperature could produce higher dipole moment in the BNT ceramic.

\subsection{Dielectric loss factor of BNT ceramics}

Figure 4 shows the dielectric loss factor of BNT ceramics against frequency for all sintering temperatures. It can be observed from the results, the dielectric loss is strongly dispersed at low frequency from $600{ }^{\circ} \mathrm{C}$ to $900^{\circ} \mathrm{C}$ sintering temperature. There is no peak found for the dielectric loss factor indicating the presence of dc conductivity. ${ }^{21-23}$ In addition, the dielectric loss factor decreases with increasing sintering temperature from $600^{\circ} \mathrm{C}$ to $900^{\circ} \mathrm{C}$. These dispersion trends also can be used to identify the formation of tungsten bronze type BNT ceramic. On the other hand, there is no more dispersion found when the sintering temperature is greater than $1000^{\circ} \mathrm{C}$. However, the inverse dielectric loss factor peak was observed from the results, and the peaks shifted to the lower frequency as sintering temperature increases. The inverse peaks and dispersions found indicate that the system do not follow the Debye model. ${ }^{24-26}$ In addition, the inverse peak exhibits two relaxations occurred in the system. The relaxation time of ceramic with low sintering temperature is difficult to define because there is no peak and presence of electrode polarization. Actually, it can be found by determining the intersection point between the dielectric

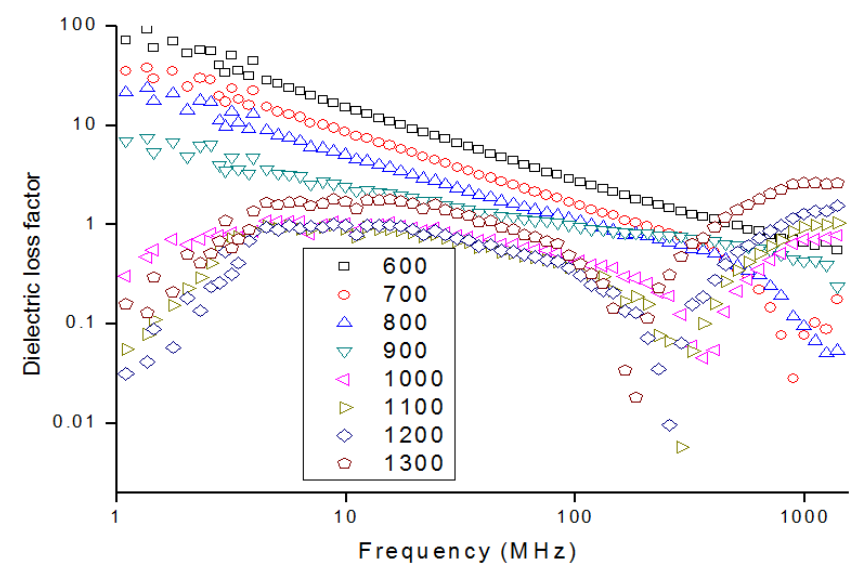

Fig. 4. Dielectric loss factor as a function of frequency for all sintering temperatures.

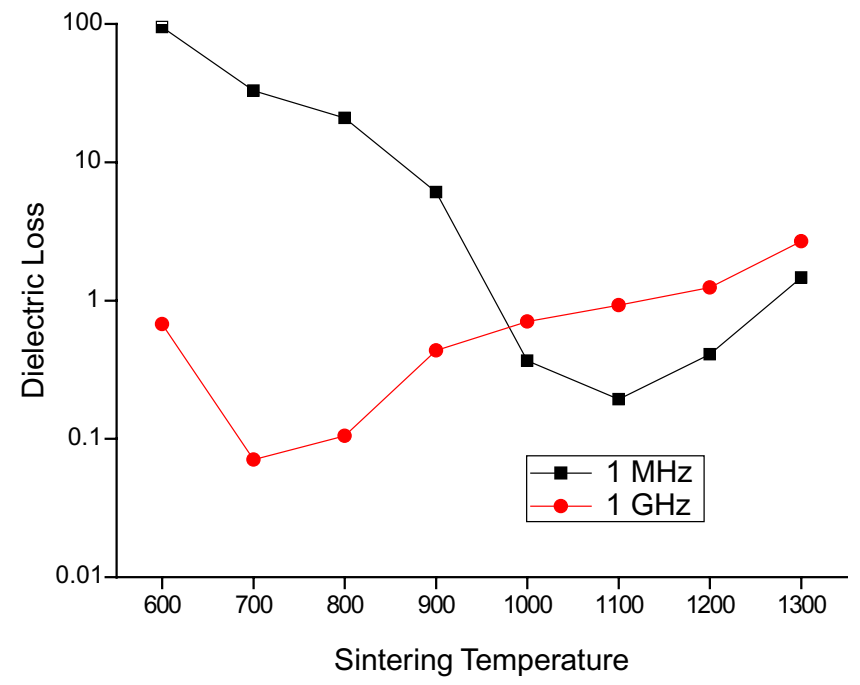

Fig. 5. Dielectric loss factor as a function of sintering temperature at frequency of $1 \mathrm{MHz}$ and $1 \mathrm{GHz}$.

permittivity and dielectric loss against the frequency, but it involved electrode and material polarization. Two relaxation times were found at high sintering temperature located at different frequency ranges. One of the relaxation time stay at lower frequency which is smaller than $10^{8} \mathrm{~Hz}$, and this is the contribution of orientation polarization. Another peak that is located at the frequency above $10^{9} \mathrm{~Hz}$, is ionic contribution. The relaxation time of high sintering temperature BNT ceramics is sintering temperature dependence due to the shifting of the inverse peak from the results. In conclusion, there are two dielectric loss factor behaviors found in the system related to the formation and sintering temperature. The dielectric loss factor as a function of sintering temperature of BNT ceramic at selected frequency was displayed in Fig. 5. The results show high dielectric loss factor at low sintering temperature led to high dissipation energy occurring in the ceramic. The lossy dielectric behavior disappeared above $1000^{\circ} \mathrm{C}$ sintering temperature when small losses were observed from the results. Figure 6 shows surface morphology of BNT ceramics at all sintering temperature that was published in previous work. ${ }^{8}$ The results shows BNT ceramics obtained round shape grains at low sintering temperature below $1000^{\circ} \mathrm{C}$ and indicating the formation not yet complete. For high sintered BNT ceramics, grains started changing to block shape and elongated at $1200^{\circ} \mathrm{C}$, and finally achieved full densification at $1300^{\circ} \mathrm{C}$. As can be seen from the dielectric constant, it increases linearly at $1 \mathrm{GHz}$ showing more crystallinity which leads to the increase in the dielectric constant.

\subsection{Complex dielectric modulus of BNT ceramics}

The electrical properties of the materials can also be represented by complex dielectric modulus, ${ }^{27-30} M^{*}=M^{\prime}-j M^{\prime \prime}$ where $M^{\prime}$ is the real part and $M^{\prime \prime}$ is the imaginary part of the 
dielectric modulus. The complex dielectric modulus is the reciprocal of complex permittivity, and can be written as follows:

$$
\begin{aligned}
& M^{\prime}=\frac{\varepsilon^{\prime}}{\varepsilon^{\prime 2}+\varepsilon^{\prime \prime 2}}, \\
& M^{\prime \prime}=\frac{\varepsilon^{\prime \prime}}{\varepsilon^{\prime 2}+\varepsilon^{\prime \prime 2}} .
\end{aligned}
$$

Basically, the dielectric modulus is used to eliminate the electrode polarization of the materials during the dielectric measurement. Figure 7 shows the real part of dielectric modulus as a function of frequency for all sintering temperatures. As can be seen from the results, the value of $M^{\prime}$ is small at low frequency for low temperature sintered ceramic, which implies that the electrode polarization is negligible. ${ }^{31}$ The $M^{\prime}$ of BNT ceramic with sintering temperature below $1000^{\circ} \mathrm{C}$ increases from low frequency region, and almost constant at high frequency region. It did not show the $\mathrm{S}$ curve in Fig. 5 because there is no constant value of dielectric permittivity at low frequency region. On the other hand, another behavior was found from the results which belong to the BNT ceramics at high sintering temperature. These

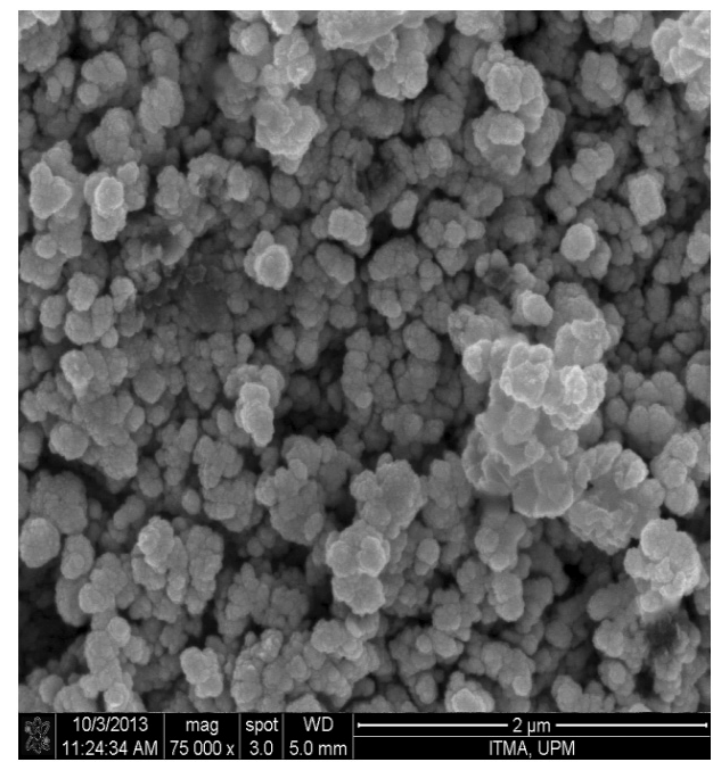

(a)

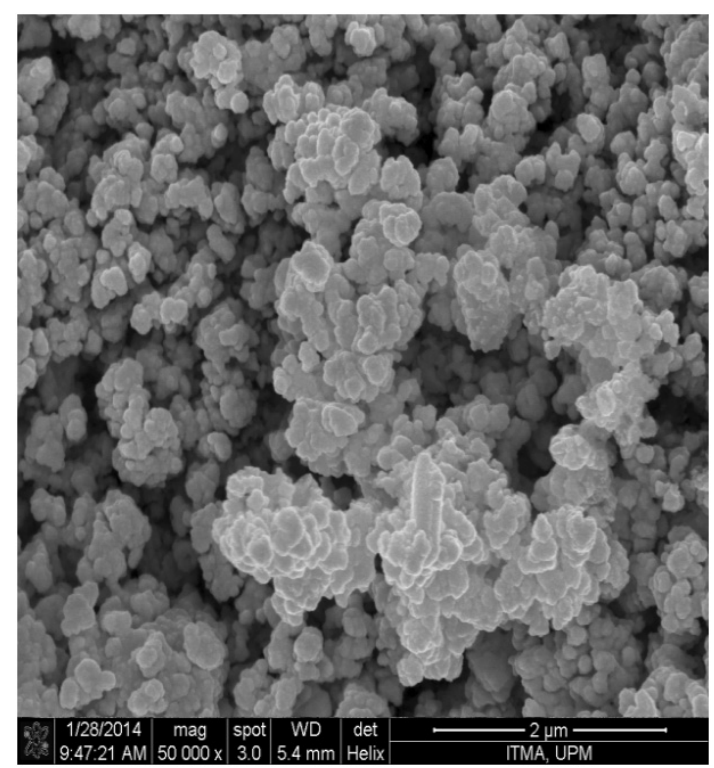

(c)

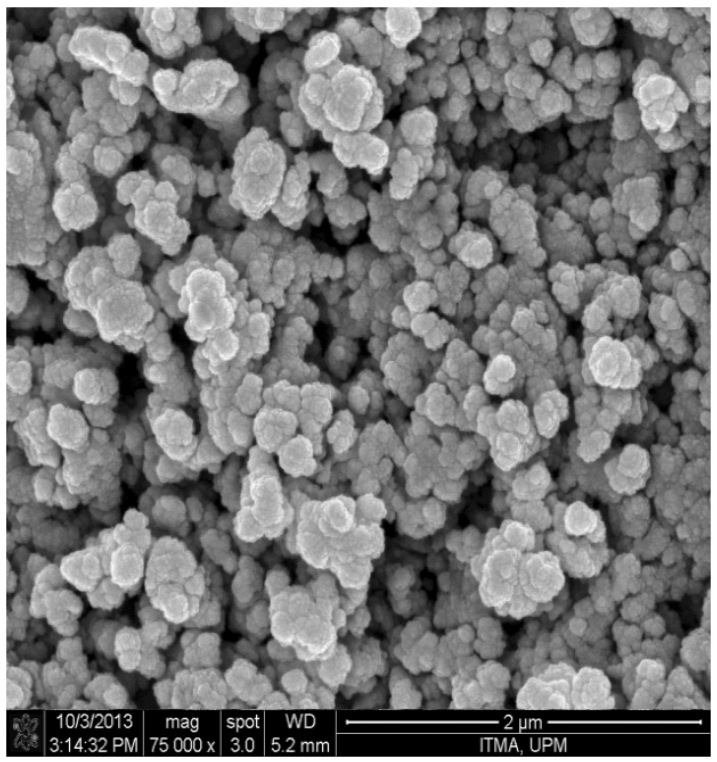

(b)

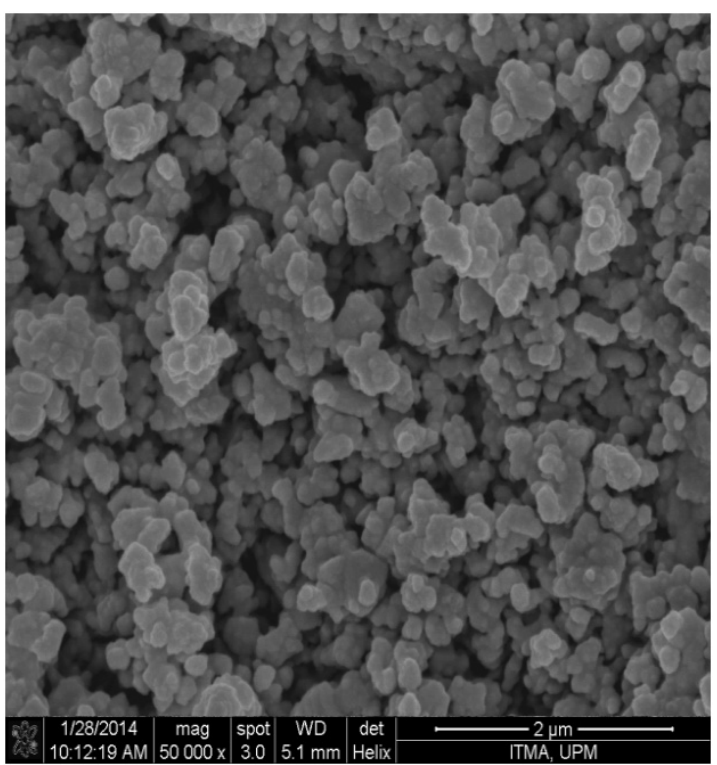

(d)

Fig. 6. Microstructure of BNT ceramic for all sintering temperatures; (a) $600^{\circ} \mathrm{C}$, (b) $700^{\circ} \mathrm{C}$, (c) $800^{\circ} \mathrm{C},\left(\right.$ d) $900^{\circ} \mathrm{C}$, (e) $1000^{\circ} \mathrm{C},(\mathrm{f}) 1100^{\circ} \mathrm{C}$, (g) $1200^{\circ} \mathrm{C}$, and (h) $1300^{\circ} \mathrm{C} .^{8}$ 


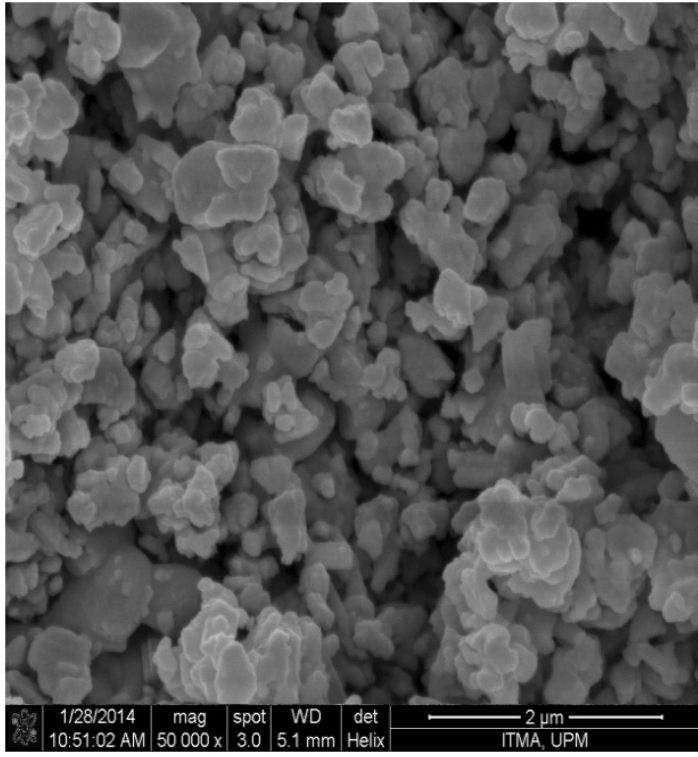

(e)

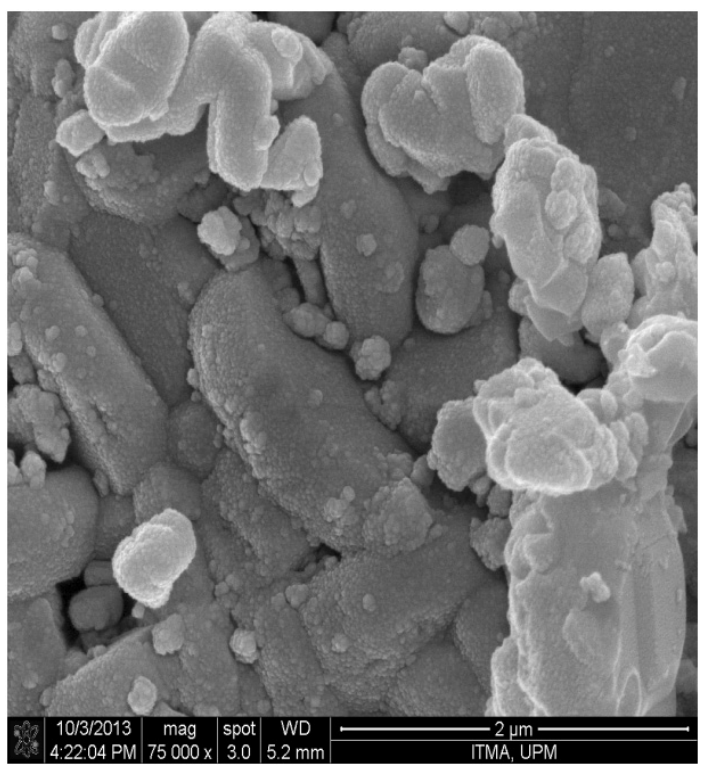

(g)

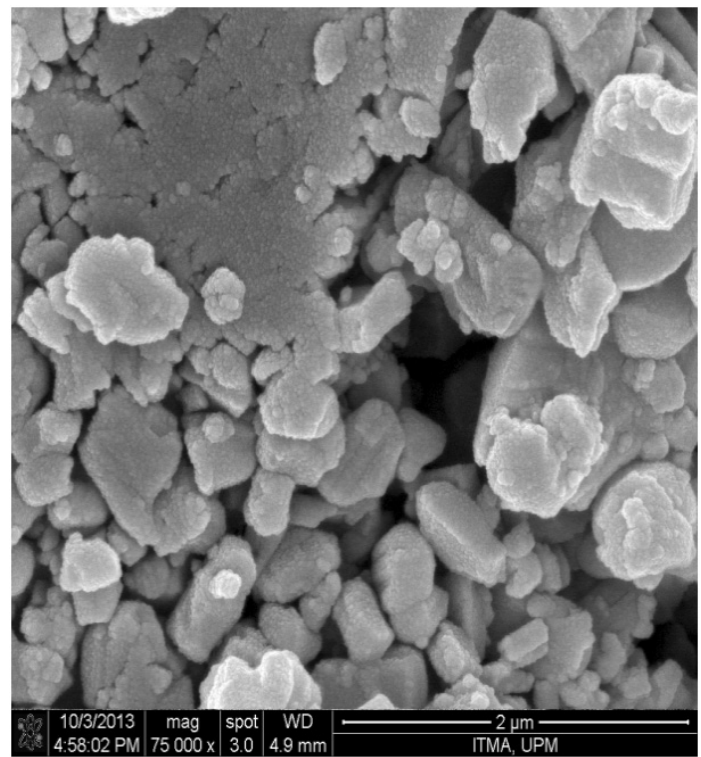

(f)

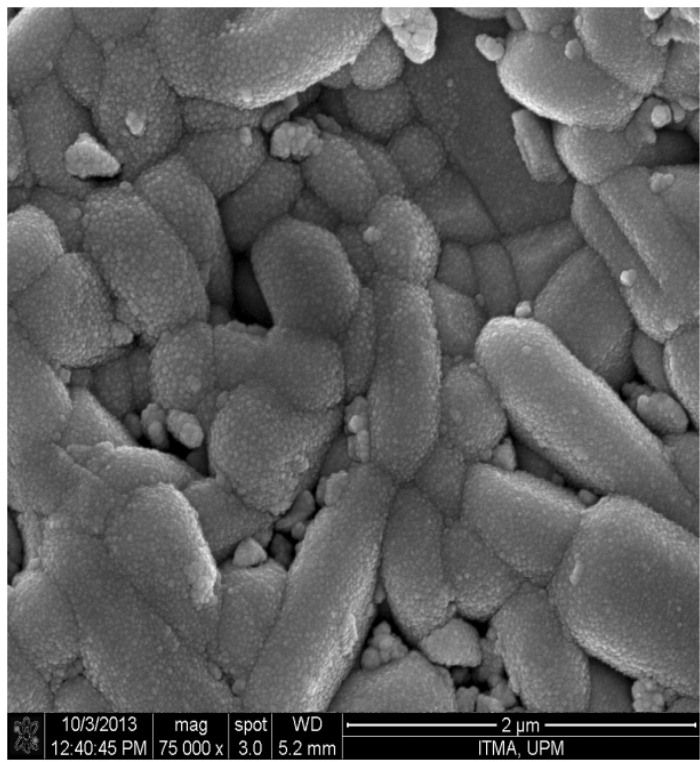

(h)

Fig. 6. (Continued)

ceramics present a constant dielectric permittivity for all frequency regions.

The imaginary part of dielectric modulus as a function of frequency for all sintering temperature is displayed in Fig. 8. Imaginary part of the dielectric modulus is more suitable to describe the relaxation behavior of the materials compared to dielectric loss and tangent loss. The results show that BNT ceramics with sintering temperature from $600^{\circ} \mathrm{C}$ to $900^{\circ} \mathrm{C}$ presented only single relaxation, but there are two relaxation in the BNT ceramic at higher sintering temperature. The relaxation frequency can be determined from the peak of the curve for each sintered ceramic. As observed in Fig. 8, the relaxation frequency of the ceramics with low sintering temperature is always present at the low frequency regime, and shifted to the lower frequency value as sintering temperature increases. This means that the ceramics underwent the long range hopping mechanism, and the charge motion is between the grain and grain boundary. For high sintering BNT ceramics, the relaxation frequency shows similar trend with increasing sintering temperature for the first relaxation. However, the relaxation frequency of the second peak of the ceramic is difficult to define since it is beyond the frequency 


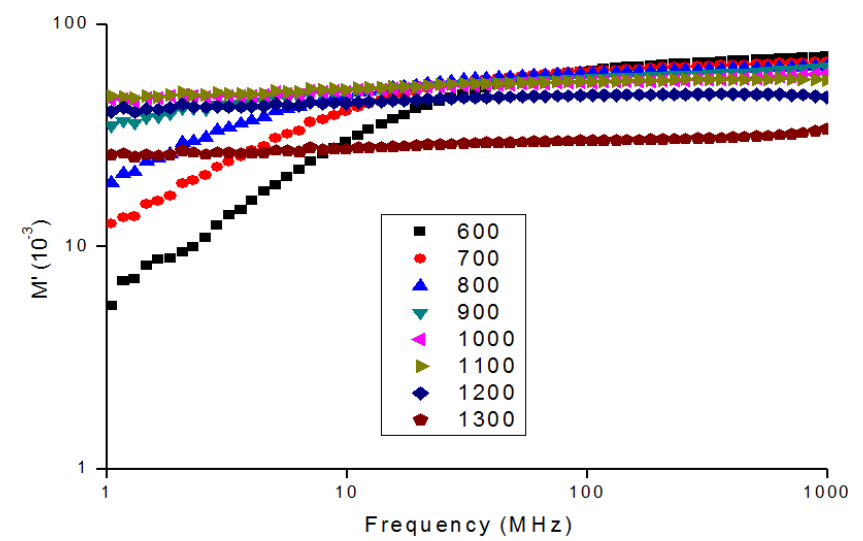

Fig. 7. Real part of dielectric modulus as a function of frequency for all sintering temperatures.

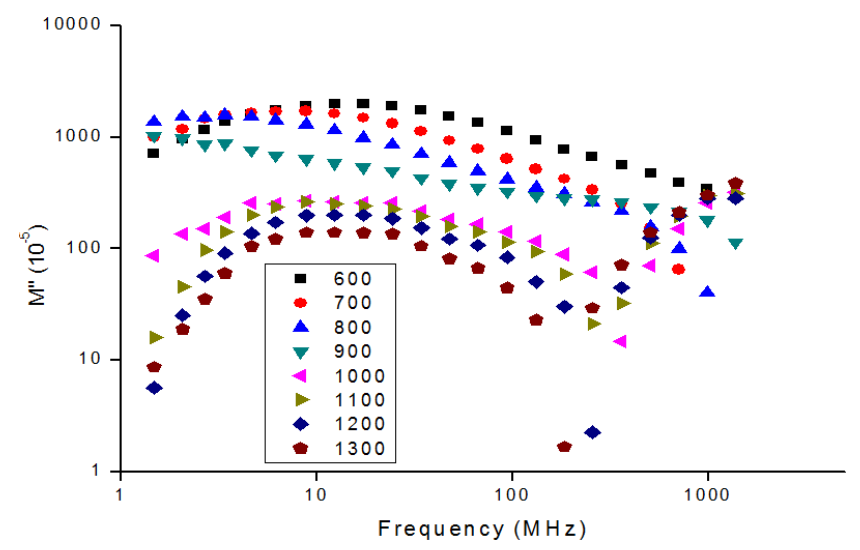

Fig. 8. Imaginary part of dielectric modulus as a function of frequency for all sintering temperatures.

applied to the sample. The second peak can be considered as the short range hopping mechanism, and the charge can only move in the localized state in the grain. The relaxation time of BNT ceramics was calculated and tabulated in Table 1. Complex plane analysis is a mathematical technique involving real and imaginary part of the complex electrical quantities of complex impedance, complex admittance, complex permittivity, and complex modulus. This is a very useful technique that can be used to evaluate the contributions of interface, grains, and grain boundary of the material with electrical response. The complex modulus plot is more effective for identifying the contribution of the grain or grain boundary due to it responses to the capacitance, and conduction mechanism for ionic and electronic relaxation. ${ }^{32}$ Figure 9 shows the complex dielectric modulus plot for all sintered BNT ceramics. For low sintered ceramics, the complex modulus spectrum shows only single semicircular arc, and this contribute to the grain of the ceramic. The semicircular arc started changing when sintering temperature increases due to the presence of $\mathrm{Nd}\left(\mathrm{TiO}_{3}\right)$ phase which might influence the grain of the sample. BNT ceramic sintered at $900^{\circ} \mathrm{C}$ shows linear shapes due to the formation of $\mathrm{Nd}_{2} \mathrm{Ti}_{2} \mathrm{O}_{7}$ phase. There seem to be one semicircular arc and a skew that can be observed at high sintering temperature. One semicircle at low frequency contributes to the grain boundary of the ceramic. It can be shown from Fig. 9 that there is a skew on the semicircular arc at high frequency for high sintered ceramics, and that is grain contribution. This shows the grain contribution of these ceramics might be very small, and the grain boundary is more dominant. However, the grain boundary can be assumed as multiple phases and impurities in the materials. It can be expected that the maximum point of the semicircular arc gives the information of conducting behavior of the material. As can be seen from Fig. 9, the lowest sintered ceramic has a maximum value of 0.02 . This also indicates greatest hopping mechanism occurring compared to other samples. However, the value of the maximum point is reduced when the sintering temperature increased. This shows the information on the transformation of semiconducting behavior to insulating behavior of the material itself. This transformation can be considered as the diffusion of neodymium ions into the system. As the melting point of $\mathrm{Nd}_{2} \mathrm{O}_{3}$ is pretty high, the interaction between the $\mathrm{Nd}^{+}$ion

Table 1. Microstructure and dielectric properties of BNT ceramic at all sintering temperatures.

\begin{tabular}{lccccccc}
\hline & & \multicolumn{5}{c}{ Dielectric properties } \\
\cline { 6 - 7 } $\begin{array}{l}\text { Sintering } \\
\text { temperature }\left({ }^{\circ} \mathrm{C}\right)\end{array}$ & $\begin{array}{c}\text { Average grain } \\
\text { size }(\mu \mathrm{m})\end{array}$ & $\begin{array}{c}\text { Density } \\
\left(\mathrm{g} \mathrm{cm}^{-3}\right)\end{array}$ & $\varepsilon^{\prime}(1 \mathrm{MHz})$ & $\varepsilon^{\prime \prime}(1 \mathrm{MHz})$ & $\varepsilon^{\prime}(1 \mathrm{GHz})$ & $\varepsilon^{\prime \prime}(1 \mathrm{GHz})$ & $\begin{array}{c}\text { Relaxation } \\
\text { time } \tau_{M}\left(\times 10^{-8}\right)(\mathrm{s})\end{array}$ \\
\hline 600 & 0.131 & 4.08 & 73.02 & 94.71 & 13.81 & 0.67 & 1.24 \\
700 & 0.185 & 3.982 & 49.69 & 32.94 & 14.77 & 0.07 & 2.44 \\
800 & 0.197 & 3.944 & 33.46 & 20.93 & 15.42 & 0.10 & 5.97 \\
900 & 0.207 & 4.076 & 24.01 & 6.08 & 15.34 & 0.44 & 10.47 \\
1000 & 0.322 & 3.907 & 20.01 & 0.37 & 16.56 & 0.71 & 1.39 \\
1100 & 0.456 & 3.941 & 19.29 & 0.19 & 17.79 & 0.93 & 1.87 \\
1200 & 0.687 & 4.251 & 22.08 & 0.41 & 21.36 & 1.24 & 1.03 \\
1300 & 0.754 & 4.572 & 35.31 & 1.47 & 29.19 & 2.68 & 1.29 \\
\hline
\end{tabular}

*Notes: The results for average grain size and density were obtained from Lee et al. ${ }^{8}$ 


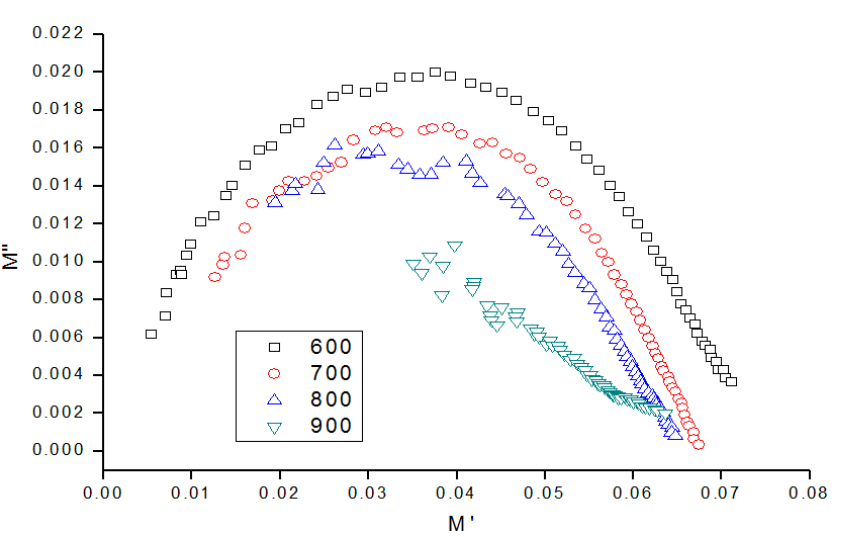

(a)

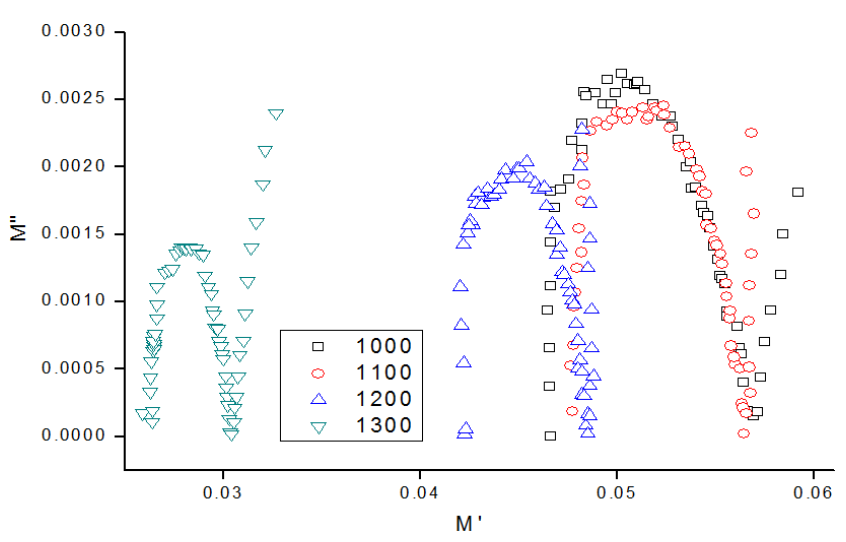

(b)

Fig. 9. Complex dielectric modulus plots for all sintering temperatures. (a) $600-900^{\circ} \mathrm{C}$ and (b) $1000-1300^{\circ} \mathrm{C}$.

and the system should occur at high temperature. In high sintered BNT ceramics, shifting behavior in complex modulus plot is normally due to the changing effect of $M^{\prime}$.

\subsection{Complex impedance plot of BNT ceramics}

Figure 10 shows complex impedance plot of BNT ceramics at different sintering temperatures. The complex impedance analysis can be used to point out the electrical properties of the materials. ${ }^{33}$ The results show a small semicircular arc for BNT ceramic sintered at $600^{\circ} \mathrm{C}$. At this low microwave frequency region, resistance of grain was defined. It could be seen from the figure that the resistance increases with sintering temperature. The trend of semicircular arc can be observed until sintering temperature of $900^{\circ} \mathrm{C}$. This indicated that the materials exhibit more conducting behavior within this temperature. The results also satisfied with the dielectric loss factor that was mentioned above that showed the presence of dc conductivity. On the other hand, the complex impedance plot for BNT ceramics sintered above $1000^{\circ} \mathrm{C}$ showed no more semicircular arc, and have similar trend to

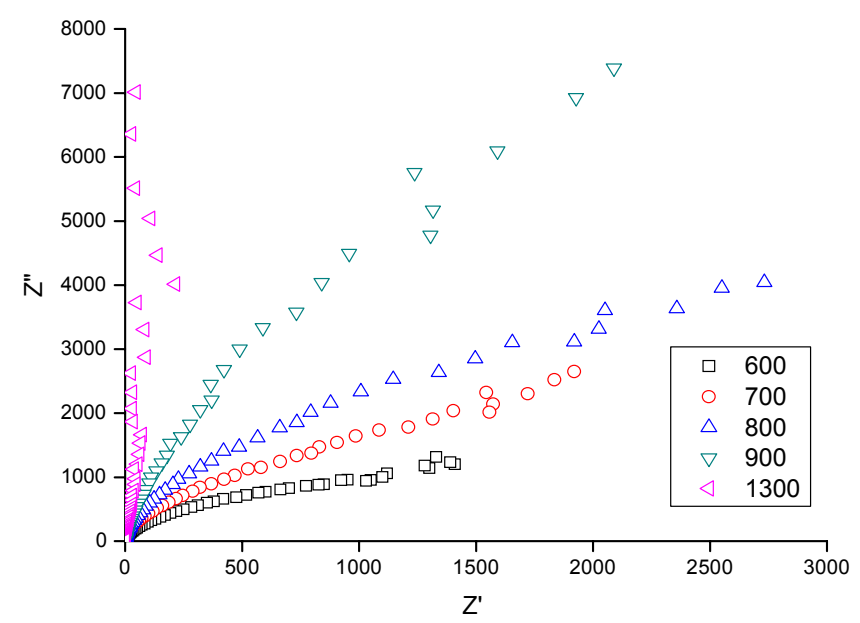

Fig. 10. Complex impedance plots for BNT ceramics.
BNT $1300^{\circ} \mathrm{C}$. The vertical increases of the graph indicated the materials have higher resistance.

\section{Conclusion}

In conclusion, the dielectric properties of BNT ceramics strongly depend on the grain size and chemical reaction inside the material. The relaxation time of low sintered BNT ceramics increased with sintering temperature. Complex dielectric modulus was utilized to investigate the properties of grain and grain boundary behavior. Conduction mechanism of BNT ceramic decreased with increasing sintering temperature. Complex impedance plot confirmed the resistance increased with sintering temperature. BNT ceramic sintered at $1300^{\circ} \mathrm{C}$ obtained highest dielectric constant at $1 \mathrm{GHz}$ that could be a promising material for microwave technology.

\section{Acknowledgments}

This research was financially supported from the Research University Grant Scheme (RUGS) Project No.: 05-02-122180RU, Universiti Putra Malaysia (UPM). The authors also acknowledged the Department of Physics, Faculty of Science, UPM and Institute of Advanced Technology (ITMA), UPM.

\section{References}

${ }^{1}$ Z. L. Huan et al., Crystal structure and microwave dielectric properties of $\left(\mathrm{Zn}_{1-x} \mathrm{Co}_{x}\right) \mathrm{TiNb}_{2} \mathrm{O}_{8}$ ceramics, J. Alloys Compd. 551, 630 (2013).

${ }^{2} \mathrm{P}$. Zhang et al., Effect of $\mathrm{CaTiO}_{3}$ addition on microwave dielectric properties of $\mathrm{NdNbO}_{4}$ ceramics as multi-function material, $J$. Alloys Compd. 581, 741 (2013).

${ }^{3}$ R. D. Guo et al., Effects of $\mathrm{Bi}_{2} \mathrm{O}_{3}$ on FMR linewidth and microwave dielectric properties of LiZnMn ferrite, J. Alloys Compd. 589, 1 (2014).

${ }^{4}$ A. Sayyadi-Shahraki et al., A new temperature stable microwave dielectric ceramic with low-sintering temperature in $\mathrm{Li}_{2} \mathrm{TiO}_{3}-$ $\mathrm{Li}_{2} \mathrm{Zn}_{3} \mathrm{Ti}_{4} \mathrm{O}_{12}$ system, J. Alloys Compd. 597, 161 (2014). 
${ }^{5} \mathrm{~L} . \mathrm{X}$. Li et al., Composite dielectrics $(1-y)\left(\mathrm{Mg}_{1-x} \mathrm{Zn}_{x}\right)_{1.8}$ $\mathrm{Ti}_{1.1} \mathrm{O}_{4}-\mathrm{yCaTiO} \mathrm{C}_{3}$ suitable for microwave applications, J. Alloys Compd. 531, 18 (2012).

${ }^{6}$ D. Thomas, A. Pulanchiyodan and M. T. Sebastian, Effect of isovalent substitutions on the microwave dielectric properties of $\mathrm{Ca}_{4} \mathrm{La}_{6}\left(\mathrm{SiO}_{4}\right)_{4}\left(\mathrm{PO}_{4}\right)_{2} \mathrm{O}_{2}$ apatite, J. Alloys Compd. 546, 72 (2013).

${ }^{7}$ C. L. Huang et al., High-Q microwave dielectrics in the $\left(\mathrm{Mg}_{1-x} \mathrm{Zn}_{x}\right)_{4} \mathrm{Ta}_{2} \mathrm{O}_{9}$ ceramics, J. Alloys Compd. 590, 494 (2014).

${ }^{8}$ C. H. Lee et al., Evolution of dielectric ceramic $\mathrm{Ba}_{6-3 x} \mathrm{Nd}_{8+2 x}$ $\mathrm{Ti}_{18} \mathrm{O}_{54} \quad(x=0.15)$ with microstructure at different sintering temperatures, Int. J. Res. Appl. Nat. Social Sci. 2(5), 37 (2014).

${ }^{9}$ C. C. Cheng et al., The effect of composition on Ba-Nd-Sm-Ti-O microwave dielectric materials for LTCC application, Mater. Chem. Phys. 79(2-3), 119 (2003).

${ }^{10}$ C. C. Cheng et al., Microwave dielectric properties of glassceramic composites for low temperature Co-firable ceramics, J. Eur. Ceram. Soc. 23(14), 2553 (2003).

${ }^{11}$ C. C. Cheng, Effects of composition on low temperature sinterable Ba-Nd-Sm-Ti-O microwave dielectric materials, J. Eur. Ceram. Soc. 24(6), 1787 (2004).

${ }^{12} \mathrm{P}$. T. Joseph et al., Effect of addition of sol-gel prepared BBS-glass on the microwave dielectric properties of $\mathrm{Ba}(\mathrm{Sm}, \mathrm{Nd}) \mathrm{TiO}_{3}$ for LTCC technology, Ferroelectrics 332, 123 (2006).

${ }^{13}$ B. P. Kumar et al., Investigation on PZT-based nanostructured functional materials, Synth. React. Inorg. Met.-Org. Nano-Met. Chem. 44(7), 991 (2014).

${ }^{14} \mathrm{D}$. Lin et al., Structure, dielectric and piezoelectric properties of $\mathrm{Ba}_{0.90} \mathrm{Ca}_{0.10} \mathrm{Ti}_{1-x} \mathrm{Sn}_{x} \mathrm{O}_{3}$ lead-free ceramics, Ceram. Int. 40(5), 6841 (2014).

${ }^{15}$ J. C. Maxwell, Electricity and Magnetism (Oxford University Press, New York, 1973).

${ }^{16} \mathrm{~K}$. W. Wagner, The distribution of relaxation times in typical dielectrics, Ann. Phys. 40, 817 (1973).

${ }^{17} \mathrm{Z}$. Wang et al., Giant permittivity and low dielectric loss of $\mathrm{SrTiO}_{3}$ ceramics sintered in nitrogen atmosphere, J. Eur. Ceram. Soc. 34 (7), 1755 (2014).

${ }^{18}$ M. Azhar Khan et al., High frequency dielectric response and magnetic studies of $\mathrm{Zn}_{1-x} \mathrm{~Tb}_{x} \mathrm{Fe}_{2} \mathrm{O}_{4}$ nanocrystalline ferrites synthesized via micro-emulsion technique, J. Magn. Magn. Mater. 360, 188 (2014).

${ }^{19} \mathrm{R}$. Ali et al., Structural, magnetic and dielectric behavior of $\mathrm{Mg}_{1-x} \mathrm{Ca}_{x} \mathrm{Ni}_{y} \mathrm{Fe}_{2-y} \mathrm{O}_{4}$ nano-ferrites synthesized by the microemulsion method, Ceram. Int. 40(3), 3841 (2014).

${ }^{20} \mathrm{R}$. Grigalaitis et al., Dielectric and magnetic properties of $\mathrm{BaTiO}_{3}-$ $\mathrm{NiFe}_{2} \mathrm{O}_{4}$ multiferroic composites, Ceram. Int. 40(4), 6165 (2014).

${ }^{21}$ G. G. Raju, Polarization and static dielectric constant, Dielectrics Electric Fields (CRC Press, 2003).

${ }^{22}$ G. G. Raju, Dielectric loss and relaxation-II, Dielectrics in Electric Fields (CRC Press, 2003).

${ }^{23}$ G. G. Raju, Dielectric loss and relaxation-I, Dielectrics in Electric Fields (CRC Press, 2003).

${ }^{24}$ P. Debye, Polar Molecules (Dover Publications, New York, 1929).

${ }^{25}$ C. J. F. Bottcher et al., Theory of Electric Polarization. Dielectrics in Time-Dependent Fields, Vol. II (Elsevier, New York, 1978).

${ }^{26}$ Y. Wang et al., Observation of the slow, debye-like relaxation in hydrogen-bonded liquids by dynamic light scattering, J. Chem. Phys. 140(10) (2014).

${ }^{27} \mathrm{P}$. Singh et al., Dielectric characterization of bismuth layered $\left(\mathrm{Bi}_{2} \mathrm{O}_{3}\right)\left(\mathrm{Na}_{x} \mathrm{Fe}_{1-x} \mathrm{O}_{3}\right)$ ceramics, Phys. B, Condens. Matter 436, 64 (2014).

${ }^{28}$ A. A. Ali and M. H. Shaaban, Electrical properties of LiBBaTe glass doped with $\mathrm{Nd}_{2} \mathrm{O}_{3}$, Solid State Sciences 12(12), 2148 (2010).

${ }^{29}$ E. Pervaiz and I. H. Gul, High frequency AC response, DC resistivity and magnetic studies of holmium substituted Ni-Ferrite: A novel electromagnetic material, J. Magn. Magn. Mater. 349, 27 (2014).

${ }^{30}$ D. D. Ramteke and R. S. Gedam, Study of $\mathrm{Li}_{2} \mathrm{O}-\mathrm{B}_{2} \mathrm{O}_{3}-\mathrm{Dy}_{2} \mathrm{O}_{3}$ glasses by impedance spectroscopy, Solid State Ion. 258, 82 (2014).

${ }^{31}$ R. S. Gedam and D. D. Ramteke, Electrical, dielectric and optical properties of $\mathrm{La}_{2} \mathrm{O}_{3}$ doped lithium borate glasses, J. Phys. Chem. Solids 74(7), 1039 (2013).

${ }^{32}$ R. N. Bhowmik and I. P. Muthuselvam, Dielectric properties of magnetic grains in $\mathrm{CoFe}_{1.95} \mathrm{Ho}_{0.05} \mathrm{O}_{4}$ spinel ferrite, J. Magn. Magn. Mater. 335, 64 (2013).

${ }^{33} \mathrm{~L}$. Pavic et al., Physical properties of $\mathrm{ZnF}_{2}-\mathrm{PbO}-\mathrm{TeO}_{2}: \mathrm{TiO}_{2}$ glass ceramics-Part III dielectric dispersion and Ac conduction phenomena, Ceram. Int. 40(4), 5989 (2014). 